\title{
Effect of Supplementation on Weight Gain of Growth Faltered under 2 Children in A Rural Area of Bangladesh
}

\author{
S TASNIM ${ }^{\mathrm{a}}$, S AFROZA ${ }^{\mathrm{b}}$, F RAHMAN $^{\mathrm{c}}$
}

\begin{abstract}
Summary:
Effect of food supplementation for different duration on weight gain of growth faltered under 2 children was assessed in this study to identify an optimum duration for supplementation.

This was a longitudinal prospective study conducted among 510 children aged 6-23 months enrolled for food supplementation from 1st November 98 to 30th May' 99 under community based food supplementation program of Bangladesh Integrated Nutrition Project (BINP). The children were assigned in 3 groups of supplementation for 30, 60 and 90 days. All children were assessed for Graduation (weight gain $500 \mathrm{gm}$ ) at assigned duration of supplementation and followed for 90 days with monthly anthropometric monitoring.
\end{abstract}

\section{Introduction}

Malnutrition has been declared as silent emergency by $\mathrm{UNICEF}^{1}$. Malnutrition is most prevalent among 6-23 months children as they are more prone to inadequate weaning practices ${ }^{2}$. In Bangladesh about 60 percent children between $6 \& 71$ months are malnourished with about $17 \%$ are wasted and $51.4 \%$ are stunted ${ }^{3}$.

Supplementary feeding programs to improve nutritional status of malnourished children are in wide use in different countries ${ }^{4}$. The impact of such programs are inconsistent and vary according to variation in target population, timing of start of intervention and duration of supplementation ${ }^{5,6}$.

a. Dr Saria Tasnim, FCPS, MMEd, Diploma in Community Epidemiology.Associate Professor (Gynae)

b. Dr Syeda Afroza, FCPS, MMEd. Professor (Paediatrics) \& Joint Director, Institute of Child and Mother Health

c. Dr Fazlur Rahman, MPhil, PhD. Director, Centre for Injury Prevention and Research, Bangladesh.

Address of Correspondence: Dr Saria Tasnim, Apartment 5/D, House No.-12, Road- 126, Sal-Amanale Apartments, Gulshan-2, Dhaka-1212, Ph-01819221096.

Received: 9 December, 2006

Accepted: 13 February, 2007
The proportion of graduation was 21.7 percent (CI 15.828.7), 48.8 percent (CI 41.1-56.6) and 80.5 percent (CI 73.4-86.4) at 30, 60 and 90 days of supplementation respectively. There was no significant difference of mean weight gain between 60 and 90 days of supplementation. There was limited impact on weight gain once graduation was achieved.

As continuing supplementation will require more cost and graduated children could maintain weight gain with family diet it is recommended to assess for graduation after 60 days instead of existing practice of 90 days supplementation in the national nutrition program and consider 60 days supplementation as an optimum duration for growth faltered under 2 children.

(J Bangladesh Coll Phys Surg 2007; 25 : 121-125)

Although the growth of malnourished child might continue with continued food supplementation there is chance of limited impact on growth once momentum of weight gain is reached ${ }^{7}$.

To mitigate the problem of malnutrition among children Government of Bangladesh implemented a community based food supplementation program for growth faltered children aged 6-23 months through Bangladesh Integrated Nutrition Project (BINP) since 1995. The criteria of growth faltering under BINP program was weight loss or not gaining weight over last two months and weight gain less than 300 grams over three consecutive monthly growth monitoring and was accepted for this study ${ }^{8}$. The target for supplementation of BINP program was to achieve $500 \mathrm{gm}$ weight gain (graduation) after 90 days of supplementation and in the absence of such gain supplementation was continued for further 90 days. $^{8}$ The present study has been done to assess the effect of different length of supplementation on weight gain and to identify an optimum duration of supplementation for growth faltered children. 


\section{Methodology}

This was a longitudinal prospective study conducted during $1^{\text {st }}$ November' 98 to $30^{\text {th }}$ May'99 on 510 children aged 6-23 months enrolled for food supplementation under BINP program in Faridpur Sadar thana (sub district) of Bangladesh. This thana had 187 Community Nutrition Centres (CNC). All the CNCs were recruited and assigned to three groups randomly to provide supplementation for either 30 , 60 (intervention group, A and B) and 90 days (control, group C). The control group CNCs followed existing program of supplementation and was required to compare the effects with intervention groups. The children at the particular $\mathrm{CNC}$ were enrolled consecutively. The supplement was a mixture of cereal consisting of rice $40 \mathrm{gms}$, pulse 20 gms, molasses 10 gms and oil 6 gms and yield approximately $150 \mathrm{Kcal}$ to each child. The food was available in dry packets processed by trained community nutrition promoters (CNPs) and was monitored extensively for quality control. The dry material was prepared into semisolid by adding measured amount of water by CNP before being fed to the children at the $\mathrm{CNC}$.

The graduation criteria in this study were weight gain of 500 gms at 30, 60 and 90 days of supplementation in group A, B and C respectively. Supplementation was discontinued among graduated children in group $\mathrm{A}$ and $\mathrm{B}$ after 30 and 60 days respectively, and in the absence of such weight gain supplementation was continued till 90 days. All children were followed up for 90days. The height and weight measurements were taken at enrolment and monthly by trained data collectors by using Salter scale with 100 gm precision and were done preferably in the morning before feeding. Height was measured by locally made wooden length board at the same time. If any child after graduation in respective group (30 and 60 days supplementation) becomes eligible for supplementation according to BINP criteria during follow up period they were free to enroll again in the supplementation program.

The ethical clearance was obtained from ethical review committee of Institute of Child and Mother Health. Using SPSS 7.5 software program statistical analyses were carried out. The nutritional status of the children expressed in $\mathrm{Z}$ scores.

\section{Results}

The Children in each group were comparable and did not differ in selected socio-demographic factors and anthropometric indices at enrolment (Table 1,2). The distribution of drop out was 10, 3 and 8 in group A, B and $\mathrm{C}$ respectively mainly because of change of residence and 3 infants died. Proportion of graduation was 21.7 percent (CI 15.8-28.7), 48.8 percent (CI 41.1-56.6) and 80.5 percent (CI 73.4-86.4) after 30, 60 and 90 days of supplementation respectively (Figure -1). Mean weight gain at 90 days among the graduated children after 60 days supplementation was not different from those received supplementation for 90 days $(p=0.92)$. Graduated children continue to gain weight without supplementation during 90 days follow up period (Table -3 ).

Table-I

Socio Demographic Characteristics of Children at Enrolment $(n=510)$

Variables

Children group

\begin{tabular}{lcccc} 
& $\mathrm{A}(\mathrm{n}=172)$ & $\mathrm{B}(\mathrm{n}=174)$ & $\mathrm{C}(\mathrm{n}=164)$ & $\mathrm{P}$ value \\
\hline Age in months at enrolment & $12.81 \pm 3.4$ & $13.12 \pm 3.2$ & $13.05 \pm 3.4$ & 0.668 \\
Birth weight, kg (Mean $\pm \mathrm{SD})$ & $2.73 \pm 0.4$ & $2.70 \pm(0.4)$ & $2.8 \pm 0.4$ & 0.063 \\
SexBoy (\%)Girl (\%) & 45.354 .7 & 46.054 .0 & 46.353 .7 & 0.0983 \\
No of Family members (Mean $\pm \mathrm{SD})$ & $5.27 \pm 1.7$ & $5.60 \pm 2.0$ & $5.46 \pm 2.1$ & 0.030 \\
Mothers' education (Year \pm SD) & $1.55 \pm 2.5$ & $1.36 \pm 2.4$ & $1.13 \pm 2.6$ & 0.317 \\
Fathers' education (Year $\pm \mathrm{SD})$ & $2.22 \pm 3.3$ & $2.07 \pm 2.4$ & $2.01 \pm 3.3$ & 0.829 \\
Monthly income & $2886.11 \pm 1153$ & $2497.37 \pm 777$ & $2686.75 \pm 958$ & 0.250 \\
\hline
\end{tabular}

$\mathrm{P}$ value reached from one way analysis of variance among 3 groups 
Table-II

Anthropometric Characteristics of Children at Enrolment $(n=510)$

Variables

Children Group

\begin{tabular}{|c|c|c|c|c|}
\hline & $A(n=172)$ & $\mathrm{B}(\mathrm{n}=174)$ & $C(n=164)$ & $\mathrm{P}$ value \\
\hline Weight at enrolment, $\mathrm{kg}($ Mean $\pm \mathrm{SD})$ & $6.88 \pm 1.30$ & $6.77 \pm 1.28$ & $6.9 \pm 1.2$ & 0.210 \\
\hline Length at enrolment, $\mathrm{cm}($ Mean $\pm \mathrm{SD})$ & $66.0 \pm 6.0$ & $66.0 \pm 6.8$ & $67.5 \pm 6.1$ & 0.048 \\
\hline Weight for age ${ }^{\mathrm{a}}($ Mean \pm SD) & $69.61 \pm 11.4$ & $67.75 \pm 10.3$ & $66.78 \pm 12.2$ & 0.073 \\
\hline Height for age ${ }^{a}($ Mean \pm SD) & $87.41 \pm 7.04$ & $88.03 \pm 5.8$ & $89.07 \pm 6.6$ & 0.069 \\
\hline W/A Z score & $-3.10 \pm 1.5$ & $-3.0 \pm 1.35$ & $-2.79 \pm 1.54$ & 0.188 \\
\hline H/A Z score & $-2.70 \pm 1.0$ & $-2.9 \pm 0.91$ & $-2.99 \pm 1.08$ & 0.029 \\
\hline
\end{tabular}

a - expressed as percentage of NCHS reference value

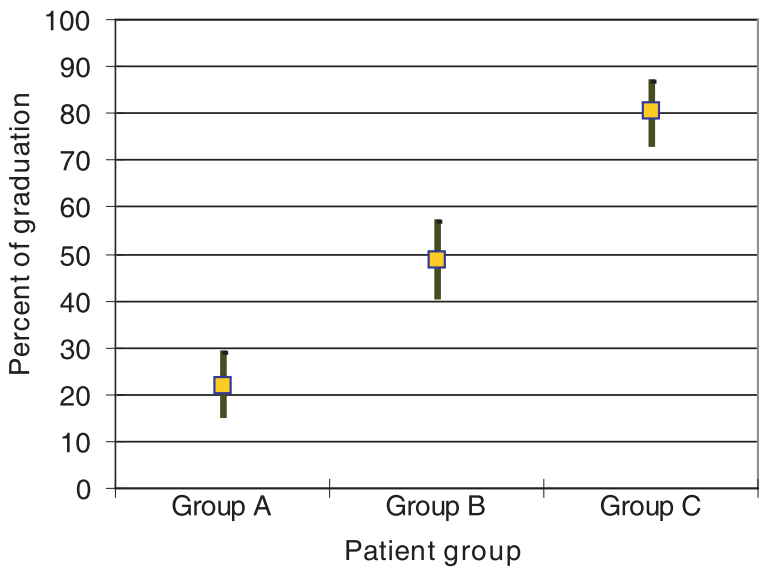

Fig.-1: Graduation rate at completion of different duration of supplementation

Table-III

Sustainability weight gain

\begin{tabular}{lccccc} 
Characteristics & \multicolumn{4}{c}{ Mean weight gain } \\
\cline { 2 - 6 } & Baseline & $30 \mathrm{~d}$ & $60 \mathrm{~d}$ & $90 \mathrm{~d}$ & P Value \\
\hline Graduated \& Supplementation for 30 days $(\mathrm{n}=37)$ & $6.06 \pm 1.32$ & $0.89 \pm .69$ & $1.1 \pm 0.92$ & $1.33 \pm 0.93$ & 0.21 \\
Graduated \& Supplementation for 60 days ( $=83)$ & $6.35 \pm 1.10$ & $0.45 \pm .38$ & $0.85 \pm .45$ & $0.96 \pm 0.67$ & 0.92 \\
Graduated \& Supplementation for 90 days ( $\mathrm{n}=154)$ & $6.92 \pm 1.28$ & $0.33 \pm .32$ & $0.66 \pm 0.43$ & $0.97 \pm 0.52$ & 0.97 \\
\hline
\end{tabular}

\section{Discussion}

The finding of this study suggests that food supplementation even for as short as 30 days could be beneficial in some children with poor nutritional status, and proportion of graduation increase with time. However, momentum of weight gain does not depend on duration of supplementation and increasing duration of supplementation from 60 to 90 days have insignificant effect on weight gain. The graduated children did not relapse back within 90 days observation period.

The study subjects were randomized appropriately as indicated by similar socioeconomic status and anthropometrics measurements. The data collectors were not aware of the study objectives and methodology. However, the investigators had no 
control over enrolment of children for supplementation and there may be some bias as has been shown that about 20.4 percent were not underweight and 32.5 percent not stunted at enrolment ${ }^{9}$. Although the household food intake and morbidity pattern of study children was not controlled during assessment of graduation there was no significant difference among them. Mean age at

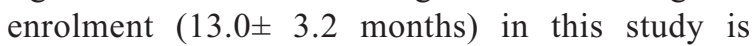
consistent with the national prevalence of under weight reported as $40 \%$ and $65 \%$ among 6-11 months and 12-23 months respectively ${ }^{10}$.

Study exploring the optimum duration of supplementation for growth faltered children consistent with similar context is scarce. In Guatemala, recovery from wasting among supplemented children aged 6-24 months increased as duration increased (attributable benefit 0.18 at 3 months to 0.25 at 6 months and 0.37 at 12 months) but larger effects were obtained during the first three months ${ }^{6}$. There is evidence that responsiveness of children to supplementary feeding with a higher yield of energy is greatest during the first 2 years with little benefit thereafter ${ }^{4}$.

Study from Jamaica among children aged 3-36 months with moderate to severe malnutrition has found that children receiving supplementation for 3 months gained significantly more in weight but the advantage was lost once supplementation was ceased 7. In a study from Indonesia, supplementary feeding over 90 consecutive days on infants aged $6-20$ months shows accelerated rate of weight gain and motor development but did not have noticeable effect on length ${ }^{11}$. It is suggested that the changes in growth rate after supplementation are more rapid and usually more marked for weight than for recumbent length ${ }^{12}$.

The Tamil Nadu Integrated Nutrition Program has shown that greater impact on prevalence of malnutrition was associated with longer duration of supplementation. ${ }^{13}$ However, a study among Bangladeshi urban slum children has revealed that during the first 3 months of intervention, the monthly weight gain of supplemented children was significantly higher than the controls, but the change was not significant in subsequent 3 months of supplementation ${ }^{14}$.
The target group in the BINP program is 6 to 24 months old and about half of children enrolled for supplementation had gain weight as per desired level of graduation within 60 days. Once graduated children could maintain weight gain with usual family diet, there was no significant difference of mean weight gain between 60 and 90 days of supplementation. However, behavior change communication component of the BINP program could have a positive synergistic effect on growth through education and awareness of parents on child care and nutrition.

\section{Conclusion and recommendation:}

Although the effectiveness of a supplementation program is assessed through nutritional impact produced and duration is a determining factor, the cost involvement is also a major concern especially for low resource countries like Bangladesh. If children are looked for graduation after 60 days instead of existing practice of 90 days and supplementation are withheld in graduated children the cost of the program can be reduced substantially without compromising the beneficial effect of supplementation. Considering the effect on weight gain it is recommended to consider 60 days supplementation as optimum duration for growth faltered under 2 children in Bangladesh.

\section{Acknowledgement}

The financial support provided by Bangladesh Integrated Nutrition project through ICDDR,B is gratefully acknowledged. Our heartfelt thanks goes to enumerators and the mothers of the children for their cooperation.

\section{References}

1. The State of the World's Children, 2000, UNICEF.

2. Calloway $\mathrm{OH}$, Murphy S, Balderstone J. Village Nurition in Egypt, Keny and Mexico; Looking Across the CRSP Project. Berkely University of California Report 1992.

3. Child nutrition survey of Bangladesh 1995-1996. Bangladesh Bureau of Statistics, Statistics division, Ministry of Planning. Government of the Peoples Republic of Bangladesh. December 1997.

4. Schroeder DG, Martorell R, Rivera J A, Marie T, Habicht J P. Age differences in the impact of nutritional supplementation on growth. J Nutr 1995; 125: 1051-59 
5. Beaton GH \& Ghassemi H. Supplementary feeding programs for young children in developing countries. Am J Clin Nutr 1982; 35: 864-916

6. Rivera J A, Habicht J P \& Robson DS. The recovery of Guatemalan children with mild to moderate wasting: factors enhancing the impact of supplementary feeding. Am J Public Health 1996; 86:1430-34.

7. Heikens G.T, Schofield W.N, Dawson S.and GranthamMcgregor S. The Kingston Project. I. Growth of Malnourished Children during Rehabilitation in the Community, Given A High Energy Supplement. European Journal of Clinical Nutrition.1989; 43: 145-160.

8. Bangladesh Integrated Nutrition Project, project proforma, 1995

9. Saria Tasnim, S Afroza, F Rahman. Socio demographic Characteristics and enrollment criteria of under 2 children in food supplementation programme under BINP. Bangladesh Journal of Child Health 2004; 28(2): 40-44.
10. Hellen Keller International, Bangladesh. The nutritional surveillance project in Bangladesh 1999. Project report 2001.

11. Husaini M.A, Karyadi L, Husaini Y.K, Sandjaja, Karyadi D and Pollitt E. Developmental effects of short-term supplementary feeding and nutritionally-at-risk Indonesian infants. J Clin Nutr 1991; 54:799-804.

12. Roche A F, Guo S and Moore W M. Weight and recumbent length from 1 to 12 months of age: reference data for 1month increments. Am J Clin Nutr 1989; 49: 599-607.

13. Chidambaram G. India Tamil Nadu Integrated nutrition project -Terminal evaluation volume I, 1989 The World Bank.

14. Fauveau C, Siddiqui M, Briend A, SilimperiD R, Begum N and Fauveau V. Limited Impact of a Target Food Supplementation Programmme in Bangladeshi Urban Slum Children. Annals of Tropical Pediatrics 1992 ; 12: 41-48. 